\title{
Dopplervelocimetria das artérias uterinas na predição de complicações em gestações de baixo risco
}

\author{
Uterine artery doppler velocimetry for the prediction of complications in low-risk pregnancies
}

Fabrício da Silva Costa ${ }^{1}$, Sérgio Pereira da Cunha ${ }^{2}$, Aderson Tadeu Berezowsky ${ }^{3}$

RESUMO

Objetivo: determinar a validade da dopplervelocimetria das artérias uterinas na predição de complicações da gestação em população de nulíparas de baixo risco. Métodos: foi conduzido estudo prospectivo que incluiu 45 pacientes primigestas, sem história de doenças crônicas. A dopplervelocimetria das artérias uterinas foi realizada entre 24 e 26 semanas, com determinação do índice de resistência (IR), índice de pulsatilidade (IP), relação S/D e a presença ou ausência de incisura na onda de velocidade de fluxo. Na análise estatística foi utilizado o teste de Mann-Whitney para amostras não paramétricas e o teste exato de Fisher foi utilizado na avaliação dos parâmentros qualitativos. Resultados: complicações da gravidez foram observadas em 12 pacientes, com quatro casos de pré-eclâmpsia, um caso de recém-nascido pequeno para a idade gestacional (PIG), um caso de PIG e parto pré-termo (PPT), três casos de PPT, um caso de centralização fetal e dois casos de mecônio espesso no líquido amniótico no momento da resolução da gestação. Notamos que o IR (mediana 0,56 x 0,68), IP (mediana 0,98 x 1,29) e a relação $\mathrm{S} / \mathrm{D}$ (mediana 2,2 × 2,9) estavam mais elevados no exame realizado entre a 24 e a $26^{\mathrm{a}}$ semana nas pacientes que apresentaram qualquer complicação da gestação e não mostrou diferença nos casos de pré-eclâmpsia ou PIG. A presença de incisura bilateral apresentou sensibilidade de 100 e 90\%, especificidade de 60,6 e 62,5\%, valor preditivo positivo (VPP) de 29,4 e 42,9\%, valor preditivo negativo (VPN) de 100 e 95,2\% para a deteç̧ão de pré-eclâmpsia ou PIG e qualquer complicação da gestação, respectivamente. A dopplervelocimetria alterada apresentou sensibilidade de 83,3 e 83,3\%, especificidade de 69,7 e 69,7\%, VPP de 33,3 e $50,0 \%$, VPN de 95,8 e 92,0\% para a detecção de pré-eclâmpsia ou PIG e qualquer complicação da gestação, respectivamente. Conclusão: índices de alta impedância e a presença de incisura bilateral nas artérias uterinas entre a $24^{\mathrm{a}}$ e a $26^{\mathrm{a}}$ semana de gestação podem ser bons indicadores de complicações gestacionais e perinatais.

PALAVRAS-CHAVE: Fluxometria por laser-doppler; Útero/irrigação sangüínea; Pré-eclâmpsia; Desenvolvimento embrionário fetal

\section{ABSTRACT}

Purpose: to determine the validity of uterine artery Doppler velocimetry for the prediction of pregnancy complications in a population of low-risk nulliparae. Subjects: a prospective study was conducted on 45 patients in their first pregnancy with no history of chronic diseases. Uterine artery Doppler velocimetry was performed between 24 and 26 weeks, with the determination of resistance index (RI), pulsatility index (PI), S/D ratio, and the presence or absence of incisure in the flow velocity wave. Data were analyzed by the Mann-Whitney test for non-parametric samples, and the Fisher exact test was used in the evaluation of the qualitative parameters. Results: pregnancy complications were observed in twelve patients, with four cases of preeclampsia, one case of small for gestational age newborn (SGA NB), one case of SGA NB + preterm delivery (PTD), three cases of PTD, one case of fetal centralization, and two cases of presence of thick meconium in the amniotic fluid at the time of pregnancy resolution. We noted that RI (median $0.56 \times 0.68$ ), PI (median $0.98 \times 1.29$ ) and S/D ratio (median $2.2 \times 2.9$ ) were higher at the examination performed between 24 and 26 weeks in patients with complications and did not differ in preeclampsia and SGA cases. The presence of bilateral incisure showed 100 and $90 \%$ sensitivity, 60.2 and $62.5 \%$ specificity, 29.4 and $42,9 \%$ positive predictive value (PPV), and 100 and $95.2 \%$ negative predictive value (NPV) for the detection of preeclampsia or SGA and of any complication of pregnancy, respectively. An altered Doppler showed 83.3 and $83.3 \%$ sensitivity, 69.7 and $69.7 \%$ specificity, 33.3 and $50.0 \%$ PPV, and 95.8 and $92.0 \%$ NPV for the detection of preeclampsia or SGA and of any complication of pregnancy, respectively. Conclusion: high impedance indices and the presence of a bilateral incisure in the uterine arteries between 24 and 26 weeks of pregnancy seem to be good predictors of pregnancy and perinatal complications.

KEYWORDS: Laser - doppler flowmetry; Uterus/blood suply; Pre-eclampsia; Embryo and fetal development

1 Professor Pesquisador do Curso de Medicina da Universidade Estadual do Ceará UECE - Fortaleza (CE) - Brasil.

2 Professor Titular do Departamento de Ginecologia e Obstetrícia da Faculdade de Medicina de Ribeirão Preto - Universidade de São Paulo - USP - São Paulo (SP) - Brasil.

3 Professor Doutor do Departamento de Ginecologia e Obstetrícia da Faculdade de Medicina de Ribeirão Preto - Universidade de São Paulo - USP - São Paulo (SP) - Brasil.

Correspondência: Fabricio da Silva Costa

Rua Eunice Weaver, 210 Casa 13 - 60.740-000 - Fortaleza-CE - Fone/Fax: (85) 3299-2824 - e-mail: fabriciouece@hotmail.com

Recebido em: 8/4/2005 Aceito com modificações em: 13/6/2005

Rev Bras Ginecol Obstet. 2005;27(6):323-30 
Introdução

As circulações feto-placentária e úteroplacentária representam um sistema de vital importância para o desenvolvimento e o bem-estar fetal. O desenvolvimento normal do feto depende de adequado suporte de oxigênio e nutrientes provenientes de eficaz perfusão do espaço interviloso através dos vasos uterinos.

Estudos nos quais se realizou biópsia do leito placentário, no terceiro trimestre da gravidez, documentaram invasão trofoblástica inadequada ou anormal como a lesão predominante em mulheres com pré-eclâmpsia, mas o mecanismo responsável por essa falha ainda não é claro ${ }^{1,2}$. Esses achados histológicos têm uma boa correlação com o aumento da resistência ao fluxo nas artérias uterinas avaliadas pela dopplervelocimetria ${ }^{3}$ e uma relação deste aumento da resistência e o subseqüente surgimento de pré-eclâmpsia ${ }^{4,5}$.

Utilizando a dopplervelocimetria como teste de rastreamento, vários estudos têm demonstrado a associação entre anormalidades na onda de velocidade de fluxo (OVF) nas artérias uterinas com o subseqüente surgimento da pré-eclâmpsia, da restrição do crescimento intra-uterino (RCIU), do descolamento prematuro da placenta (DPP) e do parto pré-termo (PPT) ${ }^{3-5}$.

Com o emprego da persistência da incisura como definição de anormalidade e realizando o exame em dois estágios da gestação $\left(20^{a}\right.$ e $24^{a}$ semanas), obtiveram-se uma especificidade de $96 \%$, valor predidivo positivo (VPP) de $28 \%$ e valor preditivo negativo (VPN) de $99,5 \%$ para a predição da pré-eclâmpsia ${ }^{6}$.

Pesquisa realizada na Finlândia ${ }^{7}$ mostrou que a dopplervelocimetria tem menor poder de predição de complicações quando aplicada a uma população de baixo risco. A dopplervelocimetria das artérias uterinas e útero-placentárias tiveram um poder maior na predição de pré-eclâmpsia e RCIU nas gestações de alto risco, identificando um grupo no qual $58,3 \%$ das pacientes desenvolveram complicações tardiamente na gestação.

Com o objetivo de selecionar mulheres com risco para o desenvolvimento de pré-eclâmpsia e RCIU, Kurdi et al. ${ }^{8}$ estudaram 1022 gestantes com realização de dopplervelocimetria das artérias uterinas entre a $19^{\mathrm{a}}$ e a $21^{\mathrm{a}}$ semana. Vinte e três por cento das pacientes apresentaram o teste alterado, sendo $12,4 \%$ com incisura bilateral. Nas mulheres com incisura bilateral, o odds ratio (OR) para o desenvolvimento de pré-eclâmpsia foi de 12,8 e nas pacientes com dopplervelocimetria normal o OR foi de 0,11. Estes autores concluem que a reali- zação de estudo dopplervelocimétrico na $20^{\mathrm{a}}$ semana da gestação pode ser usado para classificar as pacientes em grupos de alto e baixo risco para o desenvolvimento de complicações da gestação.

Um ensaio com screening ultra-sonográfico pela dopplervelocimetria das artérias uterinas para predição de complicações em mulheres de alto risco mostrou sensibilidade de $91 \%$ e especificidade de $42 \%$, utilizando como cutoff índice de resistência (IR) maior que 0,58 para a préeclâmpsia ${ }^{9}$. Para recém-nascidos pequenos para a idade gestacional (PIG) a sensibilidade foi de $84 \%$ e a especificidade de 39\%.

$\mathrm{Na}$ literatura nacional, o primeiro trabalho no qual se avaliou o fluxo útero-placentário, utilizando a dopplervelocimetria continua direcional, foi realizado por Amim Junior et al. ${ }^{10}$. Os autores notaram que a persistência da incisura diastólica se associou com o surgimento de complicações, com sensibilidade de 65,3\% e especificidade de $85,7 \%$. Quando se relaciona a dopplervelocimetria das artérias uterinas ao aparecimento do RCIU, o indice A/B mostrou boa associação, exceto quando avaliada a artéria não placentária. A persistência da incisura, de forma isolada, não se mostrou útil para a predição de RCIU.

A pré-eclâmpsia e a RCIU estão associadas a significante morbidade e mortalidade perinatal e sua prevenção permanece como importante objetivo do atendimento obstétrico. A detecção precoce de mulheres com maior risco para essas complicações, com métodos adequados de rastreamento, pode melhorar o prognóstico materno-fetal. A avaliação dopplervelocimétrica da circulação útero-placentária e feto-placentária parece ser bom método para a predição destas complicações da gestação. Neste trabalho avaliamos a evolução dos indices de impedância nas artérias uterinas, medidos por meio da avaliação dopplervelocimétrica, em quatro períodos da segunda metade da gestação, e correlacionamos os indices de impedância e as alterações da onda de velocidade de fluxo nas artérias uterinas, medidos pelo método Doppler, com o surgimento de complicações da gestação e/ou do período perinatal.

\section{Métodos}

A casuística deste estudo compreendeu 45 mulheres na sua primeira gestação, sem doenças crônicas, gestacionais ou ginecológicas, que foram atendidas no Ambulatório de Pré-Natal do Departamento de Ginecologia e Obstetrícia do Hospital das Clínicas, da Faculdade de Medicina de 
Ribeirão Preto (HCFMRP), da Universidade de São Paulo ou na rede pública municipal de Ribeirão Preto-SP e encaminhadas para a avaliação ultrasonográfica da gestação. O estudo foi aprovado pelo Comitê de Ética em Pesquisa do HCFMRP. Os exames foram realizados após explicação prévia à paciente e a assinatura do termo de consentimento livre e esclarecido.

A determinação da idade gestacional foi feita pela data da última menstruação e realização de ultra-sonografia transvaginal no primeiro trimestre da gestação, realizado na unidade de origem da paciente ou pelo pesquisador nos casos de ausência de exame anterior.

As pacientes mantiveram o acompanhamento pré-natal nas unidades de origem até o final da gestação. Foi oferecida a realização de exames extras ao protocolo nos casos de intercorrências ou por solicitação do pré-natalista. Nos casos de complicações da gestação, as pacientes foram encaminhadas para acompanhamento no Ambulatório de Pré-natal Patológico do Departamento de Ginecologia e Obstetrícia do HCFMRP.

Os critérios de exclusão do estudo foram a existência de doenças crônicas, gestacionais ou ginecológicas, o uso crônico de medicações, tabagismo, gestações múltiplas, presença de malformações fetais, abortamento e não-comparecimento aos retornos agendados.

As pacientes foram avaliadas sempre pelo mesmo observador, após micção espontânea, em decúbito dorsal com a cabeceira elevada a $45^{\circ}$. O equipamento utilizado foi da marca ATL (Advanced Technologies Laboratories, USA), modelo HDI 3000, acoplado a transdutor convexo de 2-5 $\mathrm{MHz}$ (banda larga), dotado de Doppler pulsátil, Color Doppler e Doppler de Amplitude (Power Doppler). O filtro foi fixado em $100 \mathrm{~Hz}$. O estudo dopplervelocimétrico foi realizado em quatro períodos da gestação: $18^{\mathrm{a}}$ à $20^{\mathrm{a}}$ semana, $24^{\mathrm{a}}$ à $26^{\mathrm{a}}$ semana, $28^{\mathrm{a}}$ à $30^{\mathrm{a}}$ semana e $34^{\mathrm{a}}$ à $36^{\mathrm{a}}$ semana. Os vasos avaliados foram as artérias uterinas direita e esquerda em seus ramos ascendentes. Para a realização do exame, o transdutor foi colocado no quadrante inferior lateral do abdomen e angulado medialmente, sendo utilizado para identificar a artéria uterina homolateral ao transdutor, no ponto que cruza a artéria ilíaca externa, e a amostra de volume colocada aproximadamente a $1 \mathrm{~cm}$ do ponto de cruzamento. As medidas dos índices dopplervelocimétricos foram realizadas quando se obtiveram cinco OVF similares e de qualidade satisfatória, calculados pela média das cinco ondas, e a presença ou ausência de incisura nas artérias uterinas foi observada.

Os indices de impedância utilizados foram os consagrados na literatura: IR, indice de pulsatilidade (IP) e a relação A/B (Figura 1). A presença de incisura bilateral nas artérias uterinas foi considerada como anormalidade na OVF. Foi considerada como avaliação dopplervelocimétrica alterada nas artérias uterinas, segundo os critérios de Bower et al. ${ }^{11}$, a presença de incisura bilateral com a média do IR>0,55 (percentil 50), incisura unilateral com a média do $\mathrm{IR}>0,65$ (percentil 90) ou média do IR>0,70 (percentil 95) na ausência de incisura no exame realizado entre 24 e 26 semanas.

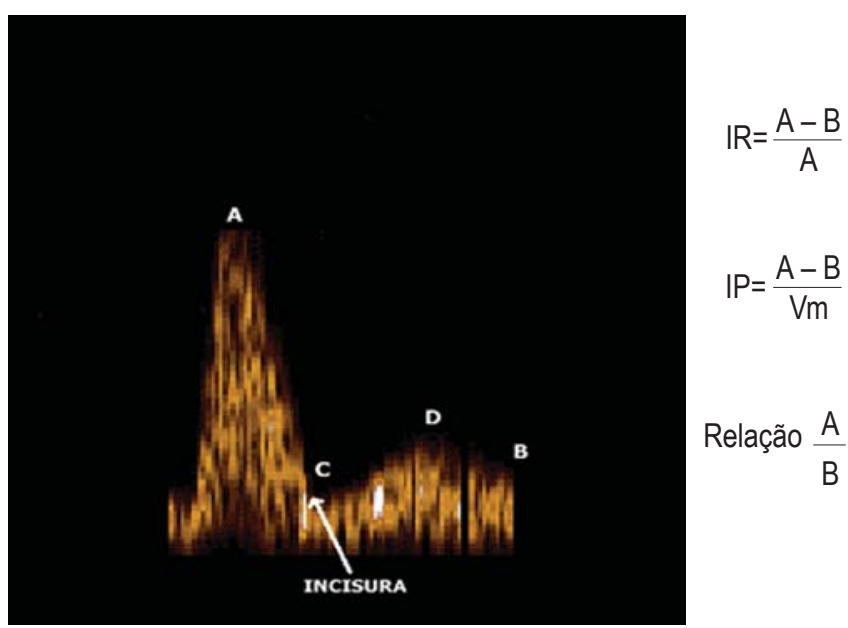

Figura 1 - Relações entre os parâmentros da onda de velocidade de fluxo (OVF) e os índices Doppler.

$\mathbb{R}$ = índice de resistência; $\mathbb{I P}=$ índice de pulsatilidade; $A=$ pico sistólico; $B$ = final da diástole; $\mathrm{Vm}=$ velocidade média; $\mathrm{C}=$ início da diástole; $\mathrm{D}=$ máximo da diástole.

As pacientes mantiveram o acompanhamento pré-natal nas unidades de origem na ausência de complicações e no HCFMRP quando qualquer complicação fosse diagnosticada, retornando para avaliação do pesquisador nos periodos préestabelecidos. Os partos foram realizados no Centro Obstétrico do HCFMRP. Os recém-nascidos foram recepcionados e acompanhados pela equipe do Departamento de Pediatria e Puericultura do referido hospital. Foram consideradas complicações perinatais a centralização cerebral do fluxo sangüineo fetal, presença de mecônio espesso no líquido amniótico no momento do nascimento e os recém-nascidos PIG. Consideramos recém-nascidos PIG aqueles que apresentaram peso ao nascimento abaixo de percentil 10 na curva de crescimento, estabelecida para a nossa população ${ }^{12}$.

As pacientes foram divididas em dois grupos conforme a evolução da gestação e o resultado perinatal. O grupo de pacientes normais foi constituído por mulheres que não apresentaram complicações da gestação ou perinatais, ao passo que o grupo de pacientes complicadas pelas mulheres que desenvolveram complicações da gestação ou 
perinatais. Foram consideradas complicações da gestação o surgimento de pré-eclâmpsia, o DPP e o PPT - antes da $37^{a}$ semana. O diagnóstico de préeclâmpsia foi feito nas pacientes que apresentaram aumento da pressão arterial PA>140/90 mmHg ou elevação de $30 \mathrm{mmHg}$ na pressão sistólica ou de $15 \mathrm{mmHg}$ na pressão diastólica após a $20^{\text {a }}$ semana da gestação, associado a edema e/ ou proteinúria (>300 mg em 24 horas).

Para análise estatística foi utilizado o teste de Mann-Whitney para amostras não paramétricas e o teste exato de Fisher foi utilizado na avaliação dos parâmentros qualitativos. Em todos os testes utilizou-se o intervalo de confiança de $95 \%(p<0,05)$.

\section{Resultados}

Foi avaliado um total de 45 pacientes no decorrer do estudo e as suas características epidemiológicas estão descritas na Tabela 1 . As complicações observadas no decorrer da gestação e/ou do período perinatal compreenderam quatro casos de pré-eclâmpsia, dois casos de recém-nascidos PIG, quatro casos de PPT (um caso apresentou PPT + PIG), um caso de centralização fetal e dois casos de presença de mecônio espesso no momento do parto. Não foi observado nenhum caso de DPP.

Tabela 1 - Características epidemiológicas das pacientes estudadas relacionadas com o estudo dopplervelocimétrico das artérias uterinas (segundo os critérios de Bower et al., 1992) 11 .

\begin{tabular}{lrrrr}
\hline Características & \multicolumn{3}{c}{ Doppler normal } & \multicolumn{3}{c}{ Doppler alterado } \\
\hline Idade (anos) & $22,0^{*}$ & \multicolumn{3}{c}{$21,3^{*}$} \\
Cor & $\mathbf{n}$ & $\%$ & $\mathbf{n}$ & $\%$ \\
$\quad$ Branca & 19 & 59,3 & 13 & 40,7 \\
$\quad$ Não-branca & 6 & 46,2 & 7 & 53,8 \\
História familiar & 7 & 28,0 & 7 & 35,0 \\
Idade gestacional no & & & & \\
parto (semanas) & $39,1^{*}$ & & $38,3^{*}$ & \\
Tipo de parto & & & & \\
$\quad$ Vaginal & 22 & 88,0 & 14 & 70,0 \\
$\quad$ Cesáreo & 3 & 12,0 & 6 & 30,0 \\
Peso ao nascimento & $3.229^{*}$ & & $3.060^{*}$ & \\
(gramas) & & & & \\
\hline${ }^{*}$ Média. & & & &
\end{tabular}

A presença de incisura bilateral nas artérias uterinas foi encontrada, no exame realizado entre a $24^{\mathrm{a}}$ e a $26^{\mathrm{a}}$ semana de gestação, em 21 casos $(46,7 \%)$, sendo unilateral em três casos $(6,6 \%)$, e estava ausente em 21 casos $(46,7 \%)$. A avaliação foi considerada alterada, segundo os critérios de Bower et al. ${ }^{11}$, no exame realizado entre a $24^{\mathrm{a}}$ e a $26^{\mathrm{a}}$ semana de gestação em 20 casos $(44,4 \%)$ e normal em 25 casos (55,6\%).

A presença de incisura bilateral nas artérias uterinas entre a $24^{\mathrm{a}}$ e a $26^{\mathrm{a}}$ semana apresentou-se fortemente associada ao surgimento de complicações na gestação. Nove pacientes apresentaram complicações da gestação, representando $75 \%$ do número total de pacientes que apresentaram qualquer complicação na gestação. Todos os casos de pré-eclâmpsia, um caso de recémnascido PIG, três casos de PPT e um caso de presença de mecônio espesso no momento do parto apresentaram essa alteração da OVF.

A presença de incisura unilateral foi observada somente em três casos. Uma gestação evoluiu normalmente, ao passo que um caso resultou em PPT com recém-nascido PIG e outro em centralização fetal, tendo como resultado recémnascido a termo sem complicações neonatais.

Entre os casos com OVF normal não ocorreu complicação da gestação. Das 21 pacientes que não apresentaram incisura nas artérias uterinas, somente um caso evoluiu com complicação no período perinatal.

A Tabela 2 mostra a associação entre a presença de complicações da gestação e/ou do período perinatal com a avaliação qualitativa da OVF das artérias uterinas. Nos 21 casos em que a incisura bilateral estava presente, quatro pacientes apresentaram pré-eclâmpsia, três apresentaram PPT, um recém-nascido era PIG e em um caso havia mecônio espesso no momento do parto. Nos três casos de incisura unilateral, tivemos um PPT somado a recém-nascido PIG e um caso de centralização fetal. Quando a incisura estava ausente, tivemos como complicação somente um caso, com presença de mecônio espesso no momento do parto.

\begin{tabular}{|c|c|c|c|}
\hline Dopplervelocimetria & $\mathrm{n}$ & Complicações & $\mathrm{n}$ \\
\hline \multirow[t]{4}{*}{ Incisura bilateral } & 21 & Pré-eclâmpsia & 4 \\
\hline & & PPT & 3 \\
\hline & & PIG & 1 \\
\hline & & Mecônio & 1 \\
\hline \multirow[t]{2}{*}{ Incisura unilateral } & 3 & PPT + PIG & 1 \\
\hline & & Centralização & 1 \\
\hline Incisura ausente & 21 & Mecônio & 1 \\
\hline
\end{tabular}

Rev Bras Ginecol Obstet. 2005; 27(6):323-30 
Quando analisamos anormalidade da OVF ou avaliação dopplervelocimétrica alterada com o surgimento de pré-eclâmpsia durante a gestação observamos associação significativa. A observação conjunta dos casos de pré-eclâmpsia e recém-nascidos PIG também está fortemente associada com a presença de incisura bilateral nas artérias uterinas ou IR elevado. A presença de qualquer complicação na gravidez mostrou-se mais freqüente quando havia anormalidade na OVF das artérias uterinas.

$\mathrm{Na}$ comparação dos resultados clínicos das pacientes com ausência ou presença de incisura nas artérias uterinas entre a $24^{\mathrm{a}}$ e a $26^{\mathrm{a}}$ semana de gestação, notamos significância estatística para pré-eclâmpsia $(\mathrm{p}<0,01)$, pré-eclâmpsia ou PIG $(p<0,01)$ e para qualquer complicação da gestação $(p<0,01)$. Também quando comparamos os resultados clínicos das pacientes com exame dopplervelocimétrico normal ou alterado nas artérias uterinas entre a $24^{\mathrm{a}}$ e a $26^{\mathrm{a}}$ semana de gestação, encontramos significância estatística para a pré-eclâmpsia $(\mathrm{p}<0,01)$, pré-eclâmpsia ou PIG $(\mathrm{p}<0,01)$ e para qualquer complicação da gravidez $(\mathrm{p}<0,01)$.

A acurácia da presença de incisura bilateral e do exame dopplervelocimétrico alterado para a definição do resultado da gestação é apresentada na Tabela 3. Usando a presença de incisura bilateral como critério de anormalidade, a sensibilidade para predição da pré-eclâmpsia e préeclâmpsia ou PIG foi de $100 \%$, porém a especificidade foi de 62,5 e $60,6 \%$, respectivamente, o que resultou em baixo VPP. Também observamos elevada sensibilidade (90\%), com discreta elevação do VPP para qualquer complicação da gestação. Quando utilizamos o IR elevado como parâmetro de anormalidade no fluxo das artérias uterinas observamos redução na sensibilidade para a detecção de pré-eclâmpsia ou PIG e qualquer complicação da gestação, com discreto aumento na especificidade e no VPP.

Tabela 3 - Acurácia da incisura bilateral e da dopplervelocimetria alterada nas artérias uterinas entre a 24 e a $26^{\mathrm{a}}$ semana de gestação na predição de complicações da gestação.

\begin{tabular}{lrrrr}
\hline & Sens. & Esp. & VPP & VPN \\
\hline $\begin{array}{lrrrr}\text { Incisura bilateral } \\
\text { Pré-eclâmpsia }\end{array}$ & 100,0 & 62,5 & 25,0 & 100,0 \\
Pré-eclâmpsia ou PIG & 100,0 & 60,6 & 29,4 & 100,0 \\
Qualquer complicação & 90,0 & 62,5 & 42,9 & 95,2 \\
Dopplervelocimetria alterada & & & & \\
Pré-eclâmpsia & 100,0 & 69,7 & 28,6 & 100,0 \\
Pré-eclâmpsia ou PIG & 83,3 & 69,7 & 33,3 & 95,8 \\
Qualquer complicação & 83,3 & 69,7 & 50,0 & 92,0 \\
\hline
\end{tabular}

Sens. = sensibilidade; Esp. = Especificidade; VPP = valor preditivo positivo; VPN = valor preditivo negativo; PIG = pequeno para a idade gestacional.

\section{Discussão}

A associação entre avaliação dopplervelocimétrica anormal e complicações da gravidez principalmente a RCIU e a pré-eclâmpsia com proteinúria e hipóxia fetal, foi observada há mais de vinte $\operatorname{anos}^{4}$. A partir deste estudo original, várias outras pesquisas realizadas na década de 80 e outras mais recentes têm relacionado alterações nas OVF das artérias uterinas, principalmente a presença de incisura bilateral, com o surgimento de complicações da gestação $0^{5,13-15}$. Baseados nesses resultados promissores, grande número de estudos foram realizados para investigar o valor preditivo da dopplervelocimetria das artérias uterinas como um teste de rastreamento na gestação, previamente ao surgimento da doença ${ }^{7}$. Entretanto, os resultados têm sido conflitantes, e, apesar de a maioria dos trabalhos mostrar a associação de alterações dopplervelocimétricas com o surgimento de doenças ${ }^{6,16-18}$, outros estudos têm falhado em demonstrar esta associação $0^{19-21}$.

No nosso meio, existem poucos estudos prospectivos nos quais se testou a validade do exame dopplervelocimétrico das artérias uterinas para a predição de complicações da gestação. Foi com essa finalidade e também para verificar o comportamento dos índices de impedância nas artérias uterinas na segunda metade da gestação, que acompanhamos longitudinalmente um grupo de gestantes, previamente normais, até a resolução da gravidez.

Os critérios adotados nos estudos da circulação útero-placentária para a obtenção das OVF têm sido distintos. Existem relatos da avaliação das artérias arqueadas e radiais ${ }^{22}$, da artéria uterina e/ou arqueada do lado placentário e contralateral $^{23}$, além da utilização da média dos valores das artérias uterinas direita e esquerda ${ }^{9}$. O estudo das artérias subplacentárias ou das artérias arqueadas em apenas um dos lados do útero não é representativo de todo o fluxo uterino. As alterações nas artérias arqueadas placentárias somente aparecem em fases mais avançadas dos processos patológicos, não permitindo diagnóstico precoce ${ }^{10}$. Em nosso trabalho adotamos a média dos valores das duas artérias uterinas, com a amostra de volume colocada a $1 \mathrm{~cm}$ do ponto de cruzamento das uterinas com as iliacas externas, proporcionando uma avaliação mais completa da perfusão uterina.

No nosso estudo, utilizamos os três indices dopplervelocimétricos para a análise das OVF, com a intenção de verificar os seus comportamentos 
na segunda metade da gestação e as correlações com o surgimento de complicações. Nas pacientes que desenvolveram qualquer complicação na gestação e no grupo que apresentou pré-eclâmpsia ou recém-nascidos PIG também observamos redução nos índices no decorrer da gestação, porém menos pronunciada que nas pacientes que não desenvolveram doenças. Kurdi et al. ${ }^{8}$, realizando avaliação semelhante, observaram que o IR é mais elevado nas pacientes que desenvolveram complicações da gestação, sendo método útil na sua predição.

O estudo dopplervelocimétrico das artérias uterinas é considerado anormal na persistência da incisura diastólica nas $O V F$ e/ou índices dopplervelocimétricos com valor elevado, após a $20^{a}$ semana, ambos caracterizando alta resistência ao fluxo. No nosso grupo de pacientes, a incisura bilateral nas artérias uterinas estava presente em 21 casos $(46,7 \%)$ e a dopplervelocimetria foi considerada alterada em 20 casos $(44,4 \%)$ no exame realizado entre 24 e 26 semanas de gestação.

Kurdi et al. ${ }^{8}$ realizando a avaliação Doppler das artérias uterinas entre a $19^{\mathrm{a}}$ e a $21^{\mathrm{a}}$ semana, notaram a presença de incisura bilateral em 12,4\% dos casos e o teste foi considerado alterado em $22,8 \%$ dos casos. Em outro estudo no qual se avaliaram pacientes de baixo e alto risco, observouse incisura bilateral em $17,6 \%$ das pacientes de alto risco e em 8,4\% das pacientes de baixo risco ${ }^{7}$, ao passo que em outras os valores foram mais altos (40,77\% dos casos) em um grupo entre a 18 e a $26^{\mathrm{a}}$ semana de gestação.

A prevalência da incisura bilateral e da dopplervelocimetria alterada na nossa amostra foi mais elevada que a encontrada na maioria dos trabalhos. O erro interoperador para a presença ou ausência de incisura já foi reportado como sendo extremamente baixo, sendo esta avaliação qualitativa facilmente reprodutivel ${ }^{24}$. Outro fato importante é que o exame dopplervelocimétrico foi realizado em diferentes idades gestacionais, nos diferentes estudos, dificultando a comparação. Nosso estudo apresenta uma característica diversa da maioria dos trabalhos publicados na literatura, pois avaliamos somente mulheres na sua primeira gestação, fato que talvez possa explicar as diferenças com os outros achados. Especula-se que a invasão trofoblástica ocorra mais tardiamente nas primigestas e, em conseqüência, a modificação do fluxo nas artérias uterinas pode ocorrer mais tardiamente se comparado com multigestas.

A sensibilidade e a especificidade do exame dopplervelocimétrico das artérias uterinas na predição de complicações da gestação já foram amplamente testadas ${ }^{25,26}$. Utilizando a persistência da incisura bilateral ou IR elevado, Bower et al. ${ }^{6}$ chegaram a uma sensibilidade de $45 \%$ com $96 \%$ de especificidade. Kurdi et al. ${ }^{8}$, utilizando a presença de incisura bilateral, relataram uma sensibilidade de $61,9 \%$ para o diagnóstico de pré-eclâmpsia, com especifiidade de $88,7 \%$, VPP de $11,1 \%$ e o VPN de 99,0\%. Já para o diagnóstico de RCIU, a sensibilidade foi de $36,8 \%$, a especificidade de $89,2 \%$, o VPP de $17,9 \%$ e o VPN de $95,7 \%$. Quando os mesmos autores utilizaram, como critério de anormalidade no exame dopplervelocimétrico, a resistência elevada, obtiveram sensibilidade de 71,4\%, a especificidade de $78,2 \%$, o VPP de $6,9 \%$ e o VPN de $99,2 \%$ para o diagnóstico de pré-eclâmpsia, ao passo que, para o diagnóstico de RCIU, a sensibilidade foi de $47,4 \%$, a especificidade de $78,7 \%$, o VPP de $12,5 \%$ e o VPN de $95,9 \%$.

Estudo mais recente mostrou que a presença de incisura bilateral na predição de préeclâmpsia tem sensibilidade de 91,0\%, especificidade de $42,0 \%$ e VPP de $37,0 \%$ e para a predição de recém-nascidos PIG, sensibilidade de 84,0\%, especificidade de 39,0\% e VPP de 33,0\% .

Comparando-se o resultado da gestação de pacientes com dopplervelocimetria anormal nas artérias uterinas que se, após normalizados o uso de $100 \mathrm{mg} /$ dia de aspirina mostra que essas pacientes, em relação a um grupo controle, apresentaram maior risco para descolamento prematuro da placenta, baixo peso ao nascimento e recém-nascido PIG, concluindo que o retardo na normalização da avaliação dopplervelocimétrica não é fenômeno inócuo ${ }^{27}$.

Nosso estudo mostrou resultados semelhantes aos da literatura. Quando consideramos como anormalidade a presença de incisura bilateral, obtivemos, para a predição de pré-eclâmpsia, sensibilidade de 100,0\%, especificidade de 62,5\%, VPP de 25,0\% e VPN de 100,0\%. Já para a predição de pré-eclâmpsia ou PIG a sensibilidade foi de 100,0\%, especificidade de 60,6\%, VPP de 29,4\% e VPN de $100,0 \%$. Quando consideramos o surgimento de qualquer complicação da gestação, a sensibilidade foi de 90,0\%, especificidade de 62,5\%, VPP de $42,9 \%$ e VPN de $95,2 \%$. A presença de IR elevado entre a $24^{\mathrm{a}}$ e a $26^{\mathrm{a}}$ semana de gestação apresentou sensibilidade e especificidade semelhantes às anteriores, com uma melhora muito discreta no VPP.

Nossos resultados confirmam os dados da literatura e demonstram a importância da realização do estudo dopplervelocimétrico das artérias uterinas mesmo em populações de baixo ris- 
co, podendo-se selecionar pacientes com alterações do fluxo útero-placentário para acompanhamento pré-natal em unidades de referência e possibilitando a utilização de medidas profiláticas.

\section{Referências}

1. Khong TY, De Wolf F, Robertson WB, Brosens I. Inadequate maternal vascular response to placentation in pregnancies complicated by preeclampsia and by small-for-gestacional age infants. Br J Obstet Gynaecol. 1986;93(10):1049-59.

2. Meekins JW, Pijnenborg R, Hanssens M, McFayden IR, van Asshe A. A study of placental bed spiral arteries and trophoblast invasion in normal and severe pre-eclamptic pregnancies. $\mathrm{Br} \mathrm{J}$ Obstet Gynaecol. 1994;101(8):669-74.

3. Olofsson P, Laurini RN, Marsal K. A high uterine artery pulsatility index reflects a defective development of placental bed spiral arteries in pregnancies complicated by hypertension and fetal growth retardation. Eur J Obstet Gynecol Reprod Biol. 1993;49(3):161-8.

4. Campbell S, Diaz-Recasens J, Griffin DR, CohenOverbeek TE, Pearce JM, Willson K, et al. New Doppler technique for assessing uteroplacental blood flow. Lancet. 1983;1(8326 Pt 1):675-7.

5. Fleischer A, Schulman H, Farmakides G, Bracero L, Grunfeld L, Rochelson B, et al. Uterine artery Doppler velocimetry in pregnant women with hypertension. Am J Obstet Gynecol. 1986;154(4):806-13.

6. Bower S, Bewley S, Campbell S. Improved prediction of preeclampsia by two-stage screening of uterine arteries using the early diastolic notch and color Doppler imaging. Obstet Gynecol. 1993;82(1):78-83.

7. Zimmermann P, Eiriö V, Koskinen J, Kujansuu E, Ranta T. Doppler assessment of the uterine and uteroplacental circulation in the second trimester in pregnancies at high risk for preeclampsia and/or intrauterine growth retardation: comparison and correlation between different Doppler parameters. Ultrasound Obstet Gynecol. 1997;9(5):330-8.

8. Kurdi W, Campbell S, Aquilina J, England P, Harrington K. The role of color Doppler imaging of the uterine arteries at 20 weeks' gestation in stratifying antenatal care. Ultrasound Obstet Gynecol. 1998;12(5):339-45.
9. Coleman MA, McCowan LM, North RA. Mid-trimester uterine artery Doppler screening as a predictor of adverse pregnancy outcome in high-risk women. Ultrasound Obstet Gynecol. 2000;15(1):7-12.

10.Amim Junior J, Lima MLA, Fonseca ALA, Bornia RBG, Chaves Netto $H$, Montenegro CAB. Dopplerfluxometria das artérias uterinas no diagnóstico da toxemia e do crescimento intrauterino retardado. J Bras Ginecol. 1988;98(7):399402.

11.Bower S, Vyas S, Campbell S, Nicolaides KH. Color Doppler imaging of the uterine artery in pregnancy: normal ranges of impedance to blood flow, mean velocity and volume of flow. Ultrasound Obstet Gynecol. 1992;2(4):261-5.

12. Matheus M, Sala MA. Crescimento intra-uterino. Evolução da altura fetal, peso do feto, da placenta e do índice placentário, na segunda metade da gestação. Rev Assoc Med Bras. 1977;23(3):88-90.

13. Kofinas AD, Penry M, Simon NV, Swain M. Interrelationship and clinical significance of increased in the uterine arteries in patients with hypertension or preeclampsia or both. Am J Obstet Gynecol. 1992;166(2):601-6.

14.Alfirevic Z, Neilson JP. Doppler ultrasonography in high-risk pregnancies: systematic review with metaanalysis. Am J Obstet Gynecol. 1995;172(5):137987.

15.Murakoshi T, Sekizuka N, Takakuwa K, Yoshizawa $\mathrm{H}$, Tanaka K. Uterine and spiral artery flow velocity waveforms in pregnancy-induced hypertension and/or intrauterine growth retardation. Ultrasound Obstet Gynecol. 1996;7(2):122-8.

16.Steel SA, Pearce JM, McParland P, Chamberlain GV. Early Doppler ultrasound screening in prediction of hypertensive disorders of pregnancy. Lancet. 1990;335(8705):1548-51.

17. Harrington KF, Campbell S, Bewley S, Bower S. Doppler velocimetry studies of the uterine artery in the early prediction of pre-eclampsia and intrauterine growth retardation. Eur J Obstet Gynecol Reprod Biol. 1991;42 Suppl:S14-20.

18.Valensise H, Bezzeccheri V, Rizzo G, Tranquilli AL, Garzetti GG, Romanini C. Doppler velocimetry of the uterine artery as a screening test for gestational hypertension. Ultrasound Obstet Gynecol. 1993;3(1):18-22.

19.Jacobson SL, Imhof R, Manning N, Mannion V, Little D, Rey E, et al. The value of Doppler assessment of the uteroplacental circulation in predicting preeclampsia or intrauterine growth retardation. Am J Obstet Gynecol. 1990;162(1):1104. 
20.Bewley S, Cooper D, Campbell S. Doppler investigation of uteroplacental blood flow resistance in the second trimester: a screening study for preeclampsia and intrauterine growth retardation. $\mathrm{Br}$ J Obstet Gynaecol. 1991;98(9):871-9.

21.North RA, Ferrier C, Long D, Townend K, KincaidSmith P. Uterine artery Doppler flow velocity waveforms in the second trimester for the prediction of preeclampsia and fetal growth retardation. Obstet Gynecol. 1994;83(3):378-86.

23.Duggan P, McCowan L. Normal ranges for Doppler flow velocity waveforms from maternal uterine and fetal umbilical arteries. Aust N Z J Obstet Gynaecol. 1993;33(2):139-41.
24.Arduini D, Rizzo G, Boccolini MR, Romanini C, Mancuso S. Functional assessment of uteroplacental and fetal circulations by means of color Doppler ultrasonography. J Ultrasound Med. 1990;9(5):24953.

25.Arduini D, Rizzo G, Romanini C, Mancuso S. Uteroplacental blood flow velocity waveforms as predictors of pregnancy-induced hypertension. Eur J Obstet Gynecol Reprod Biol. 1987;26(4):335-41.

26. Harrington K, Fayyad A, Thakur V, Aquilina J. The value of uterine artery Doppler in the prediction of uteroplacental complications in multiparous women. Ultrasound Obstet Gynecol. 2004;23(1):50-5.

27.Kurdi W, Fayyad A, Thakur V, Harrington K. Delayed normalization of uterine artery Doppler waveforms is not a benign phenomenon. Eur J Obstet Gynecol Reprod Biol. 2004;117(1):20-3. 